\title{
Avaliação do nível de segurança estrutural de pilares de concreto armado através de metodologia numérica
}

Assessment of structural safety of reinforced concrete columns through numerical methodology

\author{
F. C. Magalhães ${ }^{*} ;$ M. de V. Real2; L. C. P. da Silva Filho³; L. C. de L. Pinheiro ${ }^{1}$ \\ ${ }^{1}$ Laboratório de Estruturas e Materiais de Construção Civil, LEMCC / IFRS Campus Rio Grande, 96201-460, Rio \\ Grande-RS, Brazil \\ ${ }^{2}$ Escola de Engenharia/Universidade Federal do Rio Grande/FURG, Av. Itália km 8, Rio Grande-RS, Brasil \\ ${ }^{3}$ Laboratório de Ensaios e Modelos Estruturais, LEME / Universidade Federal do Rio Grande do Sul/UFRGS, \\ 90040-060, Porto Alegre-RS, Brasil
}

*fabiocmagalhaes@gmail.com

(Recebido em 15 de setembro de 2014; aceito em 29 de dezembro de 2014)

\begin{abstract}
O projeto de uma estrutura de concreto armado envolve uma série de variáveis de caráter aleatório que podem ser de difícil mensuração. Dentre estas variáveis, pode ser destacada a grande variabilidade inerente às propriedades mecânicas do material, sobretudo a resistência à compressão do concreto. As incertezas decorrentes do processo de produção e controle tecnológico do concreto tendem a influenciar de forma decisiva a tomada de decisões acerca da probabilidade de falha e consequente confiabilidade das estruturas. Este trabalho apresenta um modelo computacional de simulação do comportamento de pilares de concreto armado, usando para a determinação da carga de ruptura o Método dos Elementos Finitos. O modelo foi validado através da comparação com resultados experimentais presentes na literatura. Para a avaliação da segurança estrutural utilizou-se o valor do índice de confiabilidade ( $\beta$ ); obtido através dos métodos de Monte Carlo e FORM. Os resultados mostram que o modelo é capaz de simular de forma correta o comportamento da estrutura, permitindo assim inferir sobre o nível de confiabilidade destes elementos.
\end{abstract}

Palavras-chave: confiabilidade estrutural, pilares de concreto armado, Método dos Elementos Finitos.

The design of a reinforced concrete structure involves a number of random variables that can be difficult to measure. Among these variables, it can be highlighted the great variability inherent to material mechanical properties, especially the compressive strength of concrete. The uncertainties resulting from the concrete production process and quality control tend to influence critically the decision-making about the probability of failure and consequent reliability of structures. This work presents a computational model to simulate the behavior of reinforced concrete columns using the Finite Element Method to determine the column resistance. The model was validated by comparison with experimental results available in the literature. For the assessment of structural safety it was used the reliability index $(\beta)$; obtained through Monte Carlo and FORM methods. The results show that the model is able to reproduce correctly the behavior of the structure allowing us to make inferences about the level of reliability of these elements.

Keywords: structural reliability, reinforced concrete columns, Finite Element Method

\section{INTRODUÇÃO}

Diversas metodologias podem ser empregadas para a análise e o dimensionamento de pilares de concreto armado. Dentre os algoritmos usualmente empregados [1] destacam-se o Método das Diferenças Finitas, o uso da Analogia de Mohr para o cálculo dos deslocamentos transversais do eixo do pilar e o Método dos Elementos Finitos. Os dois primeiros modelos computacionais, embora de relativa simplicidade de implementação, apresentam limitações na questão das condições de contorno, deixando o programa restrito a casos particulares de pilares. Este trabalho apresenta um modelo numérico baseado no Método dos Elementos Finitos para a análise de pilares de concreto armado, uma apresentação mais detalhada do modelo pode ser obtida em Araújo [1] e Araújo [2]. 


\section{MODELO NUMÉRICO}

O princípio básico do Método dos Elementos Finitos é a discretização da estrutura a ser analisada em um número finito de pequenos elementos que são interligados entre si através de nós em suas extremidades. Os elementos são definidos como elementos de pórtico plano de dois nós com três graus de liberdade por nó $\left(\mathrm{U}_{1}, \mathrm{U}_{2}, \ldots, \mathrm{U}_{6}\right)$ e suas respectivas ações nodais $\left(\mathrm{F}_{1 \mathrm{n}}, \mathrm{F}_{2 \mathrm{n}}\right.$, ...., $\left.\mathrm{F}_{6 \mathrm{n}}\right)$, conforme apresentado na Figura 1.

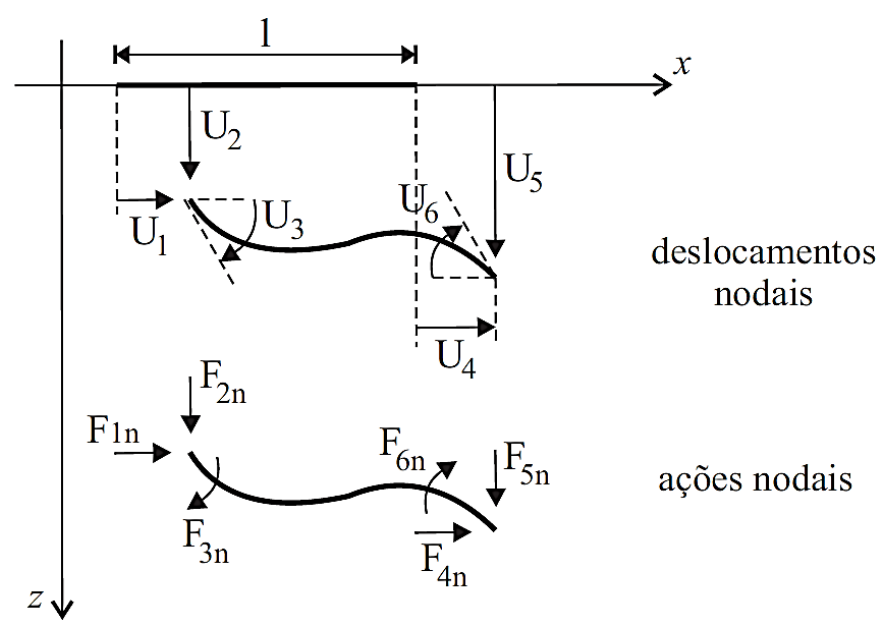

Figura 1: Ações e deslocamentos nodais de um elemento finito genérico (Adaptado: Ref.[1])

Através de uma análise linear, considerando-se a hipótese de seções planas e moderadas rotações, determina-se os deslocamentos nodais nas extremidades de cada elemento. Considerando-se uma barra sujeita a carregamento externo aplicado no plano $x$ - $z$ e as suas condições indeformada e deformada, conforme apresentado na Figura 2(a), têm-se $o$ deslocamento $u(x, z)$ de uma fibra genérica da seção situada a uma distância $z$ do eixo da barra como:

$$
u(x, z)=u_{0}(x)+u_{1}(x, z)
$$

onde $u_{1}(x, z)$ é o deslocamento provocado pela rotação da seção transversal dado por:

$$
u_{1}(x, z)=-z \theta=-z \frac{d W}{d x}
$$

Desta forma, o campo de deslocamentos no interior do elemento linear pode ser representado por:

$$
u(x, z)=u_{0}(x)-z \frac{d W}{d x}
$$

onde:

$$
W(x, z)=W(x)
$$




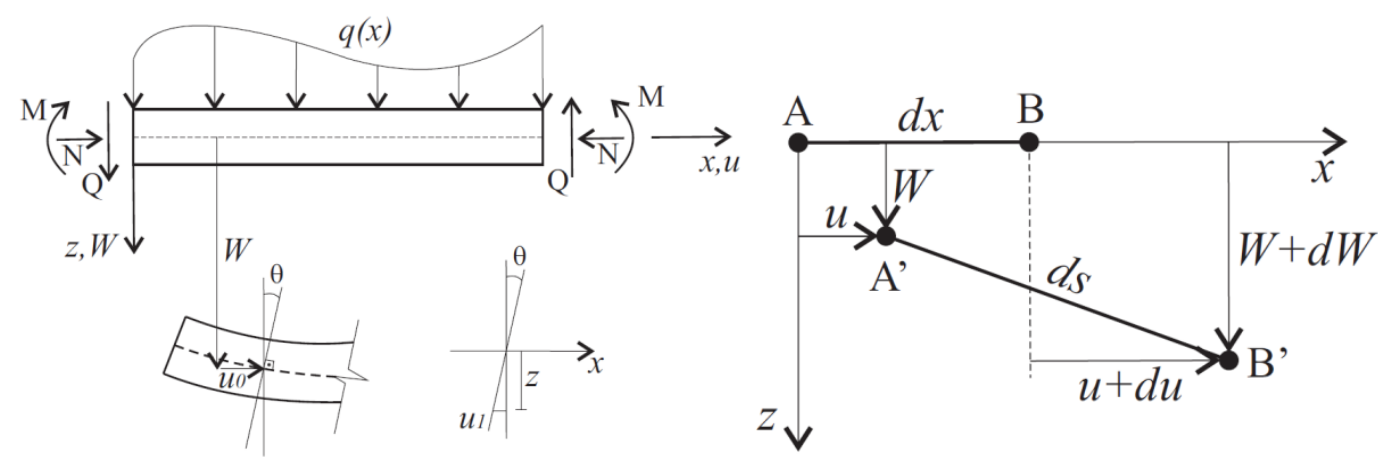

Figura 2: Esquema genérico de esforços e deslocamentos em um elemento linear (Adaptado: Ref. [1])

A partir das medidas de deslocamentos, são calculadas as deformações nos elementos discretizados. Considerando-se um segmento infinitesimal do elemento conforme esquema da Figura 2(b), têm-se as posições dos seus extremos na configuração deformada:

$$
A^{\prime}=\left\{\begin{array}{c}
x^{\prime} \\
z_{a}^{\prime}
\end{array}\right\}=\left\{\begin{array}{c}
u \\
W
\end{array}\right\} ; \quad B^{\prime}=\left\{\begin{array}{c}
x^{\prime}{ }_{b} \\
z_{b}^{\prime}
\end{array}\right\}=\left\{\begin{array}{c}
u+\frac{d u}{d x}+d x \\
W+\frac{d W}{d x}
\end{array}\right\}
$$

Sendo o comprimento final do elemento deformado $d_{S}$ dado por:

$$
d s=\sqrt{\left(x_{b}^{\prime}-x_{a}^{\prime}\right)^{2}+\left(z_{b}^{\prime}-z_{a}^{\prime}\right)^{2}}
$$

Igualando-se as Equações (5) e (6) e expandindo em série de Taylor, resulta em:

$$
d s=d x\left[1+\frac{d u}{d x}+\frac{1}{2}\left(\frac{d W}{d x}\right)^{2}\right]
$$

Considerando-se a deformação normal $\left(\varepsilon_{x}\right)$ de cada elemento dada por:

$\varepsilon_{x}=\frac{d s-d x}{d x}$

E substituindo na Equação (7) têm-se:

$\varepsilon_{x}=\frac{d u}{d x}+\frac{1}{2}\left(\frac{d W}{d x}\right)^{2}$

Substituindo na Equação (3) e derivando-se, têm-se:

$$
\varepsilon_{x}=\frac{d u_{0}}{d x}+\frac{1}{2}\left(\frac{d W}{d x}\right)^{2}-z \frac{d^{2} W}{d x^{2}}
$$

Definindo-se a deformação axial no centroide $\left(\varepsilon_{0}\right)$ e a curvatura média $(\chi)$ aproximada do elemento, respectivamente, através das Equações (11) e (12), pode-se escrever a relação entre a deformação normal os deslocamentos através da Equação (13).

$$
\begin{aligned}
& \varepsilon_{0}=\frac{d u_{0}}{d x}+\frac{1}{2}\left(\frac{d W}{d x}\right)^{2} \\
& \chi=-\frac{d^{2} W}{d x^{2}} \\
& \varepsilon_{x}=\varepsilon_{0}+z \chi
\end{aligned}
$$


Nesta análise, o termo $\left(\frac{d W}{d x}\right)^{2}$ estabelece a não linearidade da relação deformaçãodeslocamento; representando a não linearidade geométrica do problema.

Dividindo-se a seção transversal do elemento em camadas através de um modelo laminar e admitindo-se a hipótese de seções planas, calculam-se as deformações axiais em cada uma das camadas.

Para a inserção da não linearidade física dos materiais devem-se estabelecer os diagramas de tensão versus deformação que representam de forma mais adequada os materiais que compõem a estrutura. Com o intuito de comparar a solução numérica com resultados experimentais ou determinar a carga real de ruptura da estrutura, foi utilizado o diagrama tensão-deformação do concreto em compressão proposto pelo FIB Model Code [3]. O critério de ruptura da seção transversal de concreto armado é definido pelos domínios de dimensionamento, conforme a ABNT NBR 6118 [4], e os valores médios para as deformações específicas foram adotados (CEB-FIP, [5]) como $\varepsilon_{0}=2,0 \%$ e $\varepsilon_{u}=3,5 \%$. Para o aço foi admitido um comportamento elasto-plástico perfeito.

Empregando-se os diagramas tensão-deformação apropriados para os materiais, pode-se determinar em cada camada a tensão no aço e no concreto. Desta forma, os esforços solicitantes na seção transversal são dados por:

$$
\begin{aligned}
& M=\int_{A c} \sigma_{c} z d A+\sum_{i=1}^{n} \sigma_{s i} z_{s i} A_{s i} \\
& N=\int_{A c} \sigma_{c} d A+\sum_{i=1}^{n} \sigma_{s i} A_{s i}
\end{aligned}
$$

onde $A_{s i}$ é a área da armadura da camada $i$ e $n$ é o número de camadas da seção.

O esforço cortante $V$ é obtido por equações de equilíbrio, desprezando-se as deformações cisalhantes. Através do Princípio dos Trabalhos Virtuais (PTV), define-se a equação integral de equilíbrio do elemento linear, sendo considerada a condição necessária e suficiente para o equilíbrio do elemento a verificação da Equação (16)Erro! Fonte de referência não encontrada.:

$$
\delta \boldsymbol{W}_{E X T}=\delta \boldsymbol{W}_{I N T}
$$

onde $\delta \boldsymbol{W}_{E X T}$ e $\delta \boldsymbol{W}_{I N T}$ são, respectivamente, o trabalho virtual externo e interno no elemento genérico analisado.

Obtendo-se o campo de deslocamentos (Equação (2)) em um ponto genérico e determinandose as deformações e tensões correspondentes, utiliza-se o PTV para estabelecer um sistema de equações algébricas na forma:

$$
\mathbf{F}=\mathbf{K} \mathbf{U}
$$

onde $\mathbf{F}$ é o vetor de ações nodais aplicadas na estrutura; $\mathbf{U}$ é o vetor com os deslocamentos nodais e $\mathbf{K}$ é a matriz de rigidez global da estrutura.

O sistema de equações definido pela Equação (17) representa o comportamento de um material no regime não linear. Esta não linearidade decorre da não linearidade do vetor de forças nodais $\mathbf{F}$, que é função dos deslocamentos $\mathrm{F}(\mathbf{U})$, porém, a matriz de rigidez da estrutura, $\mathbf{K}$, também depende do estado de deformação da estrutura $\mathbf{K}(\mathbf{U})$. Desta forma, faz-se necessária a utilização de algum método iterativo para a análise de uma estrutura de concreto armado, considerando o comportamento não linear do material e as não linearidades geométricas do sistema. No presente trabalho o método iterativo quase-Newton BFGS é utilizado para a obtenção da convergência dos deslocamentos nodais e consequente, definição da estabilidade da estrutura para o carregamento considerado. Verificada a convergência dos deslocamentos 
fazem-se incrementos no carregamento externo e repete-se o processo iterativo até que se verifique a ruína da estrutura. Esta ruína pode ocorrer por instabilidade do equilíbrio ou por ruptura da seção transversal. O primeiro caso é detectado pela impossibilidade de convergência dos deslocamentos, enquanto que o segundo é verificado quando os esforços solicitantes não são equilibrados no estado limite último.

O processo de implementação do método dos elementos finitos utilizados no presente trabalho pode ser descrito, resumidamente, conforme segue:

- Caracterização geométrica da estrutura, tais como, o número de nós, número de elementos finitos e as coordenadas dos nós com referência a um sistema global;

- Caracterização das propriedades mecânicas dos materiais, tais como dimensões dos elementos, disposição e seção transversal das armaduras, resistências baseadas nos diagramas de tensão-deformação;

- Identificação das cargas nodais e cargas distribuídas ao longo de cada elemento finito;

- Identificação das condições de contorno do sistema;

- Montagem do vetor de cargas $\mathbf{F}$ e da matriz de rigidez $\mathbf{K}$ com base nas condições de contorno;

- Solução iterativa do sistema de equações não lineares.

No presente trabalho os pilares são modelados como pórticos planos, com dois nós e três graus de liberdade por nó. O modelo de carregamento e a discretização da estrutura em elementos finitos é apresentada na Figura 3. O sistema de equações lineares, devido às não linearidades física e geométrica, é resolvido iterativamente através do método quaseNewton do tipo BFGS. As cargas são aplicadas em pequenos incrementos, até a ocorrência de ruptura em uma seção do pilar ou instabilidade. Cada simulação determina uma carga de ruptura, assim, têm-se um conjunto de resultados de rompimentos em função da variabilidade dos dados de entrada preestabelecidos.
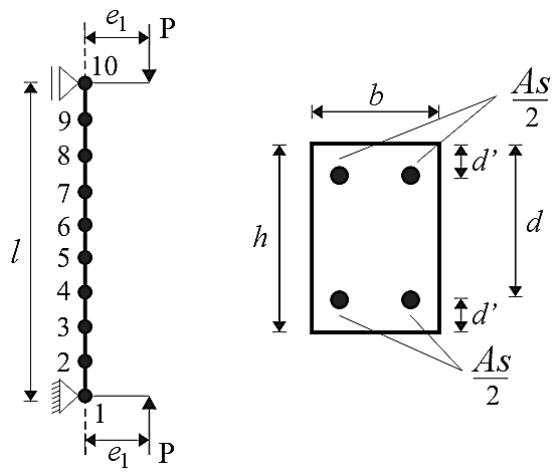

Figura 3: Geometria da seção, modelo de carregamento e discretização do eixo do pilar em elementos finitos

Conforme já relatado, o processo de dimensionamento de uma estrutura de concreto é por si só, uma tarefa que envolve uma série de fatores de caráter aleatório. Existem incertezas no valor do carregamento e de seu efeito, nas dimensões e prumos dos elementos estruturais, na resistência dos materiais e, também, nos modelos de dimensionamento propostos pelas normas.

Desta forma, a resposta da estrutura a um determinado carregamento apresenta-se como uma função de diversas variáveis aleatórias que afetam seu desempenho. Por consequência, a própria resposta da estrutura será também uma variável aleatória [6].

Neste sentido, a análise probabilística de uma estrutura surge como forma de verificar o efeito da dispersão dos parâmetros de entrada do projeto no comportamento final do sistema, tornando-se uma ferramenta de análise da confiabilidade alcançada no projeto de uma estrutura [7].

O Método de Monte Carlo envolve a geração aleatória de variáveis com distribuição de probabilidade conhecida (ou assumida) para simular o comportamento de determinado evento. Esta metodologia consiste na realização de inúmeras simulações, baseadas em um conjunto de 
parâmetros variáveis de entrada cujo modelo de distribuição de probabilidade é previamente estabelecido. $\mathrm{O}$ conjunto de resultados individuais obtidos permite a realização de uma análise estatística, possibilitando a determinação das características de distribuição de probabilidades das variáveis de resposta do sistema. A Figura 4 esquematiza o processo de funcionamento do método de Monte Carlo.

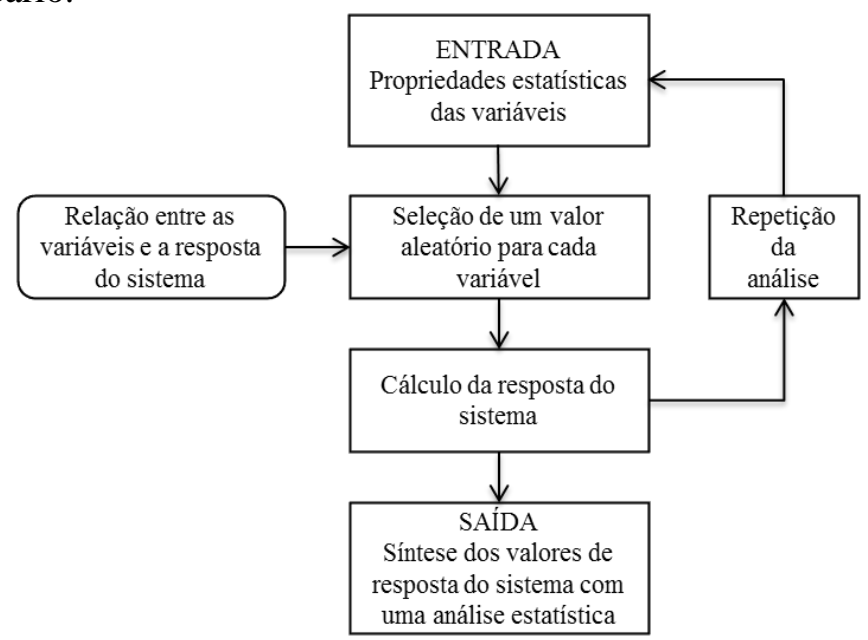

Figura 4: Esquema de funcionamento do método de Monte Carlo

A utilidade do método de Monte Carlo, na avaliação do desempenho estrutural, pode-se dar para a avaliação estatística da resposta do sistema ou para o cálculo da probabilidade de desempenho insatisfatório ou falha. No último caso a probabilidade de falha é calculada através da razão entre os casos de desempenho insatisfatório e o número total de simulações realizadas. O presente trabalho faz uso da metodologia de Monte Carlo para a avaliação estatística da resposta do sistema modelado, conforme apresentado nas próximas seções.

No caso dos pilares, foi admitido o momento fletor atuando proporcionalmente à carga axial aplicada, ou seja, com a excentricidade $e$ constante. Em termos de excentricidade relativa $(e / h)$ pode-se definir a função estado limite (função de desempenho) conforme a equação (18).

$$
g(\boldsymbol{X})=R-S=\left[P^{2}+\left(\frac{P . e}{h}\right)^{2}\right]^{\frac{1}{2}}-\left\{(G+Q)^{2}+\left[(G+Q) \frac{e}{h}\right]^{2}\right\}^{\frac{1}{2}}
$$

Onde $X$ é o vetor das variáveis básicas do problema (carga de ruptura e carregamentos); $P$ é a carga de ruptura do pilar obtida via simulação para uma excentricidade; $G$ e $Q$ são, respectivamente, as cargas permanentes e variáveis atuantes no pilar.

Na situação de excentricidade constante adotada, a carga de ruptura do pilar $(P)$ e as cargas atuantes $(G$ e $Q)$ não são correlacionadas, permitindo o tratamento em separado. Esta situação torna possível a obtenção dos parâmetros da carga de ruptura (resistência do pilar), via processo de simulação de Monte Carlo e o cálculo da confiabilidade, via outro critério de análise; por exemplo, o método FORM [8].

\section{VALIDAÇÃO DO MODELO NUMÉRICO}

Para a verificação mais precisa do erro obtido a partir do uso do modelo, foram realizadas análises comparativas dos resultados oriundos do modelo numérico com ensaios experimentais realizados por diversos autores. Este processo visa demostrar a capacidade do modelo utilizado na análise do comportamento de pilares, incluindo as não linearidades física e geométrica. Os pilares estudados são birrotulados, submetidos à flexo-compressão normal, cuja representação é apresentada na Figura 5. 

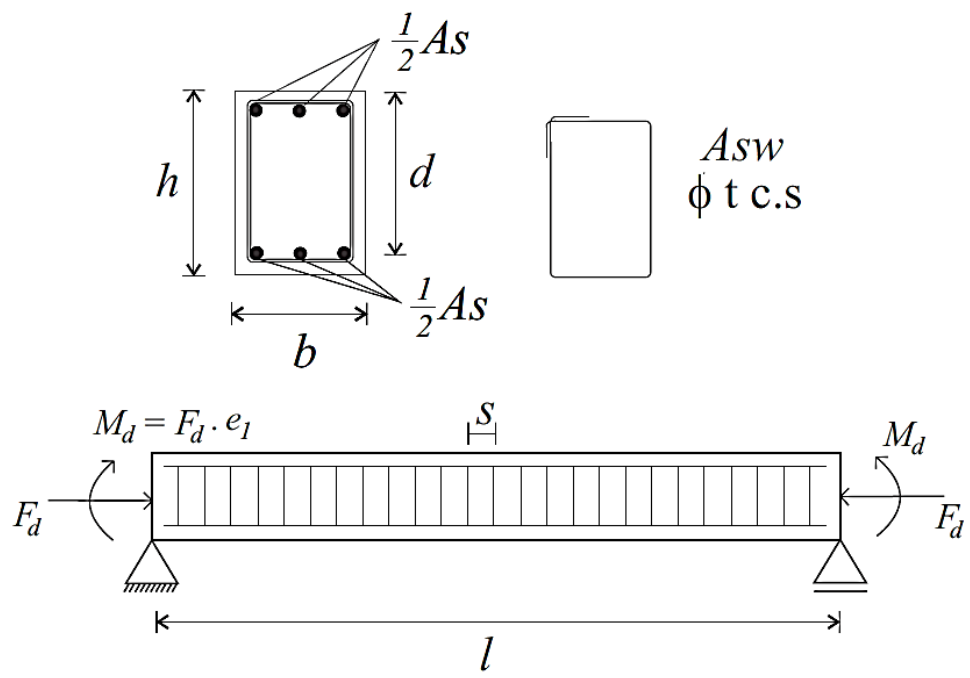

Figura 5: Características dos pilares utilizados na validação do modelo

Foram comparados os resultados experimentais apresentados por Claeson e Gylltoft [9], Goyal e Jackson [10], Melo [11], Kim e Lee [12] e Kim e Yang [13] cujo detalhamento pode ser obtido por Magalhães [15]. Foram analisados 76 resultados, permitindo estabelecer parâmetros da razão entre as cargas de ruptura experimental e do modelo $\left(p=\mathrm{P}_{\mathrm{U}, \mathrm{EXP}} / \mathrm{P}_{\mathrm{U}, \mathrm{MOD}}\right)$. Verificou-se que a relação apresentou uma média igual a 1,012 e desvio padrão igual a 0,097.

A Figura 6 apresenta o histograma obtido para os 76 pilares analisados, bem como o gráfico de aderência da razão $p$ em relação à distribuição Normal de probabilidades. Submetendo os valores de $p$ ao teste de Kolmogorov-Smirnov [14] pode-se comprovar a aderência ao modelo teórico de Gauss, uma vez que a distância máxima entre os valores de distribuição de probabilidades acumuladas dos dados e a curva Normal (Dmáx) foi inferior ao limite considerado adotando um nível de significância de 95,0 \% . Pode-se perceber uma boa simetria dos resultados em torno da média, apresentando valores tanto abaixo quanto acima do valor experimental; fornecendo um bom indício de um modelo não tendencioso.
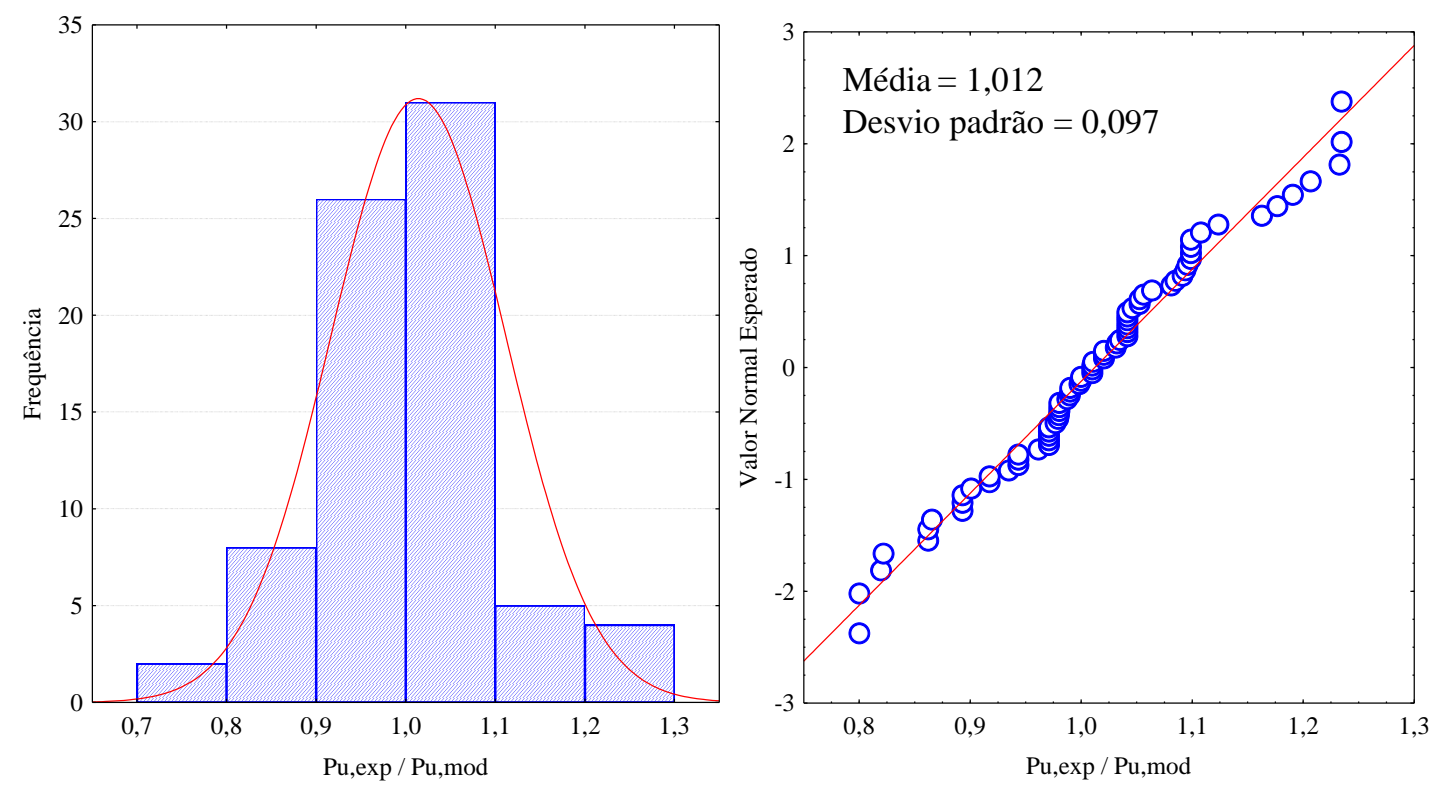

Figura 6: Histograma da relação $p=P_{U, E X P} / P_{U, M O D}$ para os pilares analisados e aderência à curva Normal 


\section{RESULTADOS DAS SIMULAÇÕES}

Com o intuito de verificar a influência da resposta no comportamento dos pilares de cada uma das propriedades considerada foram realizadas 500 simulações de Monte Carlo. Este número demostrou ser suficiente para garantir a convergência estatística dos resultados analisados. Deve-se alertar que a cada nova simulação os parâmetros de entrada eram gerados de forma aleatória e que os pilares foram levados à ruptura pelo incremento gradativo do carregamento aplicado. Os valores correspondem a pilares de concreto com $f_{c k}=30 \mathrm{MPa}$, seção transversal $40 \times 40 \mathrm{~cm}$, taxa geométrica de armadura de $0,8 \%$ e de índice de esbeltez igual a 26 , detalhadamente apresentados na Ref. [15].

O número de 500 simulações apresentou-se como suficiente para a convergência estatística do valor esperado da carga de ruptura e do seu desvio padrão. A Figura 7 apresenta a convergência do valor esperado e do desvio padrão da carga de a carga de ruptura dos pilares analisados.
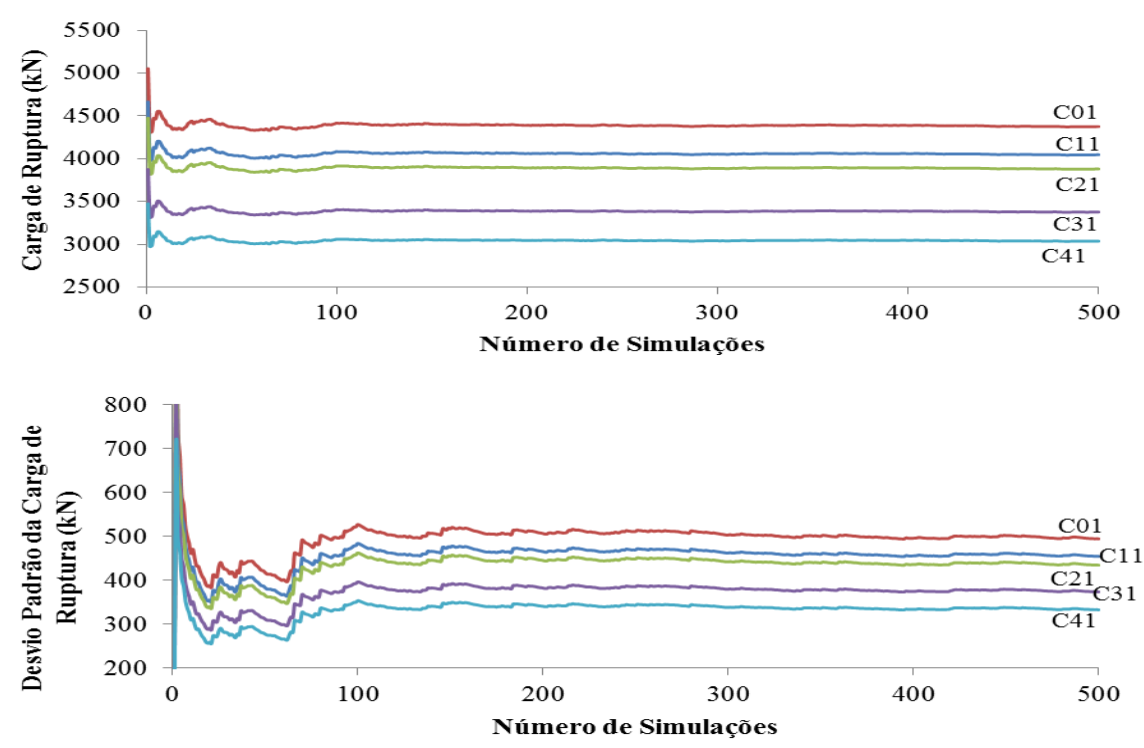

Figura 7: Convergência estatística do valor esperado e desvio padrão da carga de ruptura de pilares

A distribuição de probabilidades da carga de ruptura dos pilares analisados foi submetida ao teste de Kolmogorov-Smirnov [14], apresentando ajuste satisfatório na possibilidade de aproximação desta através de uma curva de distribuição Normal. A Figura 8 apresenta um exemplo de histograma da carga de ruptura dos pilares e o a curva de distribuição Normal ajustada aos dados. 

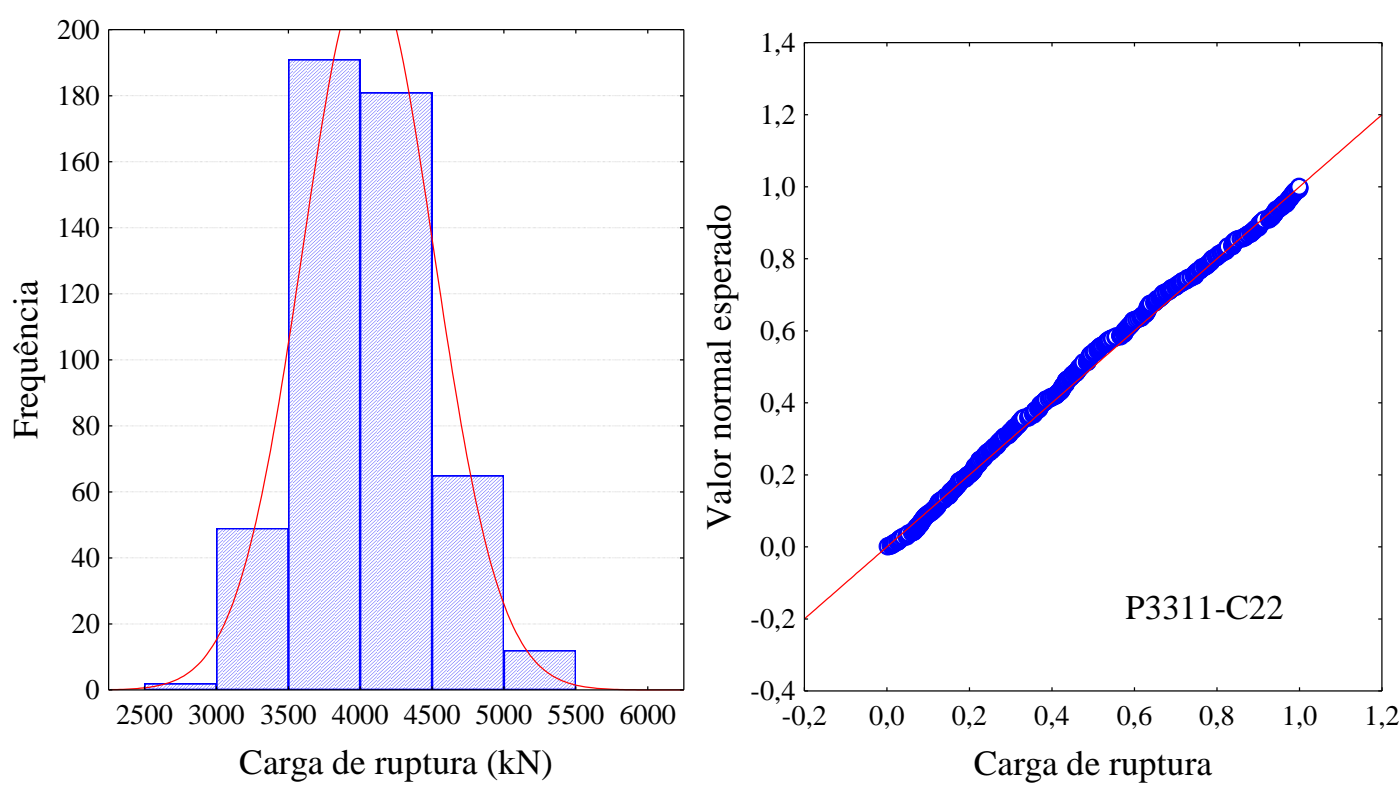

Figura 8: Histograma da carga de ruptura dos pilares e aderência à curva Normal

A convergência estatística do índice de confiabilidade pode ser verificada na Figura 9, onde pode-se observar que o número de 500 simulações foi suficiente para a estabilização dos resultados.
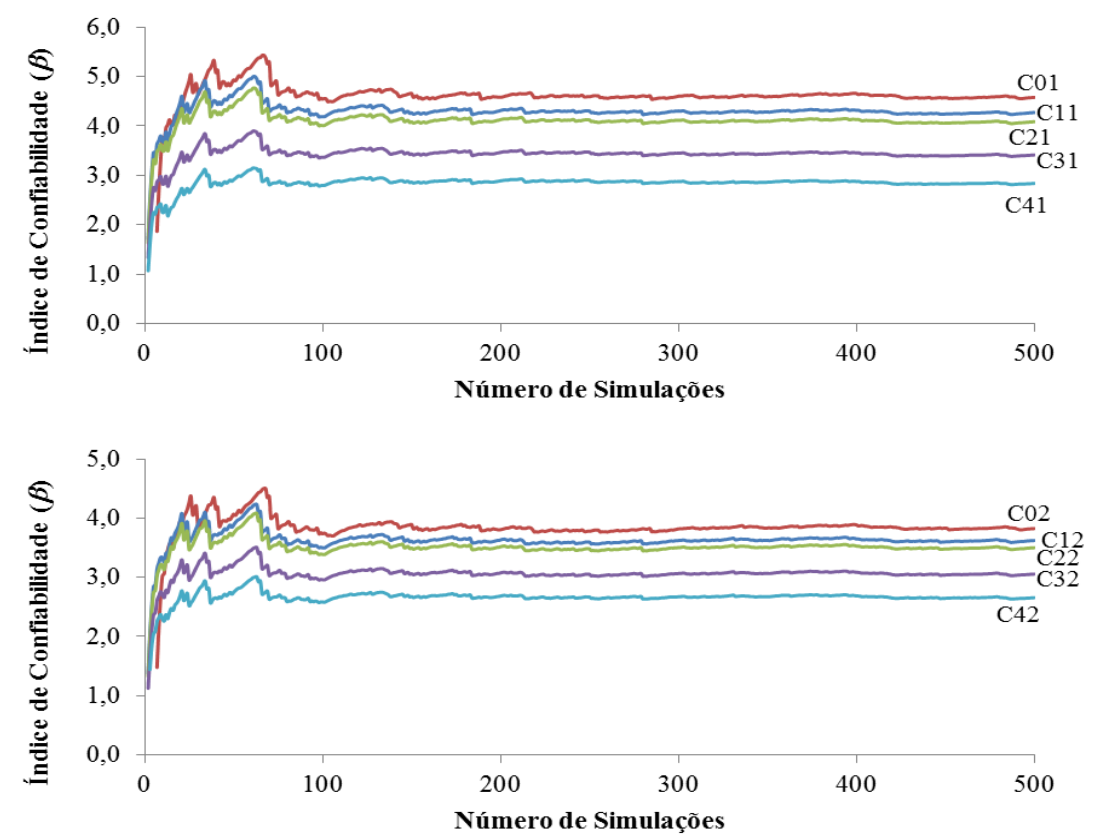

Figura 9: Exemplo de convergência estatística do índice de confiabilidade dos pilares analisados

\section{CONCLUSÃO}

O presente trabalho apresentou um modelo numérico baseado no método dos Elementos Finitos, em conjunto com o método de Monte Carlo, para a análise do comportamento estrutural de pilares de concreto armado. As não linearidades física e geométrica foram consideradas e o modelo foi validado, através da comparação com resultados experimentais de distintos autores. $\mathrm{O}$ modelo demostrou grande capacidade de simular o comportamento destes elementos estruturais e consequente determinação da carga de ruptura, sendo útil na avaliação do nível de segurança das peças analisadas. 


\section{REFERÊNCIAS BIBLIOGRÁFICAS}

1. Araújo JM de. Pilares esbeltos de concreto armado: Algoritmos para análise e dimensionamento. $1^{\text {a }}$ ed. FURG, Rio Grande-RS, 1993.

2. Araújo JM de. Curso de concreto armado. $3^{a}$ ed. Dunas, Rio Grande-RS, 2014.

3. FÉDÉRATION INTERNATIONALE DU BÉTON. FIB (CEB-FIP) Model Code 2010: First complete draft. Bulletin 55, v.1. Lausanne, Switzerland, Mar, 2010.

4. Associação Brasileira de Normas Técnicas. NBR 6118: Projetos de estruturas de concreto Procedimento. Rio de Janeiro, 2014.

5. Comité Euro-internacional du Beton. CEB-FIP Model Code 1990: Design Code. London, 437p. 1993.

6. Real MV. Análise probabilística de estruturas de concreto armado, sob estado plano de tensão, através do método dos elementos finitos. Tese (Doutorado em Engenharia), PPGEC/UFRGS. Porto AlegreRS, 2000.

7. Paliga CM. Análise probabilística de vigas de concreto armado recuperadas à flexão, através do Método de Monte Carlo utilizando um modelo de elementos finitos. Tese (Doutorado em Engenharia), PPGEC/UFRGS, Porto Alegre-RS, 2008.

8. Gomes HM. Técnicas de Avaliação da Confiabilidade em Estruturas de Concreto Armado. Tese (Doutorado em Engenharia), PPGEC/UFRGS, Porto Alegre-RS, 2001.

9. Cleason C, Gylltoft K. Slender high-strength concrete columns subjected to eccentric loading. Journal of Structural Engineering. 1998 Mar; 124:233-240.

10. Goyal BB, Jackson N. Slender concrete columns under sustained load. Journal of the Structural Division. 1971 Nov;97:2729-2750.

11. Melo CEL de. Análise experimental e numérica de pilares birrotulados de concreto armado submetidos à flexo-compressão normal. Tese (Doutorado em Engenharia), ENC/FT/UnB, BrasíliaDF, 2009.

12. Kim JK, Lee SS. The behavior of reinforced concrete columns subjected to axial force and biaxial bending. Engineering Structures. 2000;23:1518-1528.

13. Kim JK, Young JK. Buckling behaviour of slender high-strength concrete columns. Engineering Structures. 1995;17(1):39-51.

14. Ang A. H-S, Tang WH. Probability concepts in engineering planning and design. Volume II: Decision, risk, and reliability. New York, John Wiley \& Sons, 1984.

15. Magalhães FC. A problemática dos concretos não conformes e sua influência na confiabilidade de pilares de concreto armado. Tese (Doutorado em Engenharia) UFRGS, Porto Alegre-RS, 2014. 